\title{
Challenges for semilocal density functionals with asymptotically nonvanishing potentials
}

\author{
Thilo Aschebrock, ${ }^{1}$ Rickard Armiento, ${ }^{2}$ and Stephan Kümmel ${ }^{1, *}$ \\ ${ }^{1}$ Theoretical Physics IV, University of Bayreuth, D-95440 Bayreuth, Germany \\ ${ }^{2}$ Department of Physics, Chemistry and Biology (IFM), Linköping University, SE-58183 Linköping, Sweden
}

(Received 22 June 2017; published 21 August 2017)

\begin{abstract}
The Becke-Johnson model potential [A. D. Becke and E. R. Johnson, J. Chem. Phys. 124, 221101 (2006)] and the potential of the AK13 functional [R. Armiento and S. Kümmel, Phys. Rev. Lett. 111, 036402 (2013)] have been shown to mimic features of the exact Kohn-Sham exchange potential, such as step structures that are associated with shell closings and particle-number changes. A key element in the construction of these functionals is that the potential has a limiting value far outside a finite system that is a system-dependent constant rather than zero. We discuss a set of anomalous features in these functionals that are closely connected to the nonvanishing asymptotic potential. The findings constitute a formidable challenge for the future development of semilocal functionals based on the concept of a nonvanishing asymptotic constant.
\end{abstract}

DOI: 10.1103/PhysRevB.96.075140

\section{INTRODUCTION}

The paramount decision to be made when using Kohn-Sham (KS) density functional theory (DFT) [1,2] for physical, chemical, or biological applications is the choice of the approximation used for the universal exchange-correlation (xc) functional $E_{\mathrm{xc}}[n]$. A variety of approximations is available, sometimes classified according to Jacob's ladder [3,4] of DFT, reaching from the basic local functionals to constructs of increasing sophistication. The high-rung functionals nowadays achieve an accuracy that rivals the one of higher-order wavefunction-based methods [5]. However, many questions of practical relevance require functionals of the lower rungs for reasons of computational cost. These semilocal density functionals, which only depend on the electron density $n(\mathbf{r})$ and its spatial derivatives, e.g., $|\nabla n(\mathbf{r})|$, can provide an overall reasonable accuracy for $E_{\mathrm{xc}}$. Yet their functional derivatives, i.e., the corresponding xc potentials, typically completely miss important features of the exact xc potential [6-15]. Among them are, e.g., the particle-number discontinuity [16,17] and step structures or steepening effects [8,18-23] that enforce [24], e.g., the principle of integer preference. Particle-number discontinuities and potential step structures and steepenings are mathematically different properties, but they are closely related to each other $[8,17]$. Also the asymptotic features of the exact exchange [25-28] and xc [6,29] potential are not reproduced at all by standard semilocal approximations.

It became clear that these omitted features play a decisive role, e.g., in the description of charge transfer [20,30,31] and ionization $[8,17,18,32,33]$. Attempts have been made to model such features directly into semilocal xc potentials [34-45], partially also with an additional (nonlocal) eigenvalue dependence, e.g., as done by Gritsenko et al. (GLLB) [46] and Kuisma et al. [47].

In past years, the Becke-Johnson (BJ) model potential [36] and various modifications thereof $[41,48,49]$ have sparked interest in this respect by showing improved atomic-shell structure, polarizabilities, atomic and molecular properties, and band gaps closer to experimental values [36,39,48,50].

\footnotetext{
*stephan.kuemmel@uni-bayreuth.de
}

Two of the present authors have discussed that one of its key features is to effectively mimic "nonlocal" exchange features in the asymptotic behavior of the potential caused by the particle-number discontinuity by means of having a nonzero limiting value far away from a finite system [39].

However, model potentials are only of limited usefulness. Since they lack a corresponding xc energy, they cannot be used in applications that require energies, and not being functional derivatives [51,52] also renders them useless for propagating the time-dependent Kohn-Sham equations [53,54]. Furthermore, they are problematic from a formal perspective: directly modeling the $\mathrm{xc}$ potential sidesteps the original derivation of the KS equations as variational equations over the energy, and thus forgoes much of the formal framework of KS DFT. This limitation was resolved by the derivation of a semilocal energy functional, AK13 [55], designed to yield as its functional derivative a potential that shares the key features with the $\mathrm{BJ}$ model, in particular the asymptotically nonvanishing potential with a system-dependent limiting value.

The overall appeal of BJ, AK13, and derived methods is clear: including features in the exact xc potential missing from other functionals bears the promise of computational results closer to higher-order methods at the low computational expense of a semilocal functional. To some degree, the various modifications of the BJ approach and AK13 have delivered on this promise $[39,48,50]$. Thus, it may seem pertinent to ask why these methods are not more widely used. In applying AK13 and BJ to broader sets of systems, and in our attempts at improving the properties of AK13, we have identified a set of anomalies, most of which are more or less directly connected to the key property of the asymptotically nonvanishing potential. These anomalies pose a clear problem to broader adoption of functionals of this kind and present a serious challenge to their further development. The purpose of the present paper is to bring these issues to light, both as a warning against a too undiscriminating use of the present realizations of this type of methods and with the hope to inspire further development to resolve these issues.

The paper is organized as follows. First, in Sec. II, we review the AK13 and BJ functionals and their key features. In Sec. III, we discuss issues that appear when AK13 is applied to noninteger particle-number systems in the context 
of ensemble DFT. Sections IV and VI summarize findings presented elsewhere on energies and energetics of AK13 and the issue of divergent potentials along nodal surfaces. In Sec. VII, we discuss the behavior of AK13 and the BJ model in external electrical fields. Section VIII focuses on difficulties that can arise when evaluating these potentials, stemming from the numerical representation of the KS orbitals. Finally, Sec. IX gives our summary and conclusions. Hartree atomic units are used throughout this paper.

\section{AK13 AND BJ MODEL REVIEW}

The AK13 functional [55] is of the standard generalizedgradient approximation (GGA) form for exchange (x) functionals, [56] i.e.,

$$
E_{\mathrm{x}}^{\mathrm{GGA}}=A_{\mathrm{x}} \int n^{4 / 3} F(s) d^{3} r,
$$

parametrized by the reduced density gradient,

$$
s=\frac{|\nabla n|}{2 \gamma n^{4 / 3}},
$$

where $A_{\mathrm{x}}=-3 / 4(3 / \pi)^{1 / 3}$ and $\gamma=\left(3 \pi^{2}\right)^{1 / 3}$. Nevertheless, it is uniquely different from other GGAs. Its foremost feature is that its potential, as usually given by the functional derivative

$$
\begin{aligned}
v_{\mathrm{x}}^{\mathrm{GGA}}= & A_{\mathrm{x}} \frac{4}{3} n^{1 / 3}\left[F(s)-s \partial_{s} F(s)\right. \\
& +\frac{3}{4}\left(\frac{u}{s^{3}}-\frac{t}{s^{2}}\right) s \partial_{s} F(s) \\
& \left.+\left(1-\frac{3}{4} \frac{u}{s^{3}}\right) s^{2} \partial_{s}^{2} F(s)\right],
\end{aligned}
$$

with the semilocal quantities

$$
t=\frac{\nabla^{2} n}{4 \gamma^{2} n^{5 / 3}} \quad \text { and } \quad u=\frac{\nabla n \cdot \nabla|\nabla n|}{8 \gamma^{3} n^{3}},
$$

typically approaches a positive system-dependent constant outside a finite system. This is achieved by a divergence of the enhancement factor $F(s) \propto s \ln (s)$ as $s \rightarrow \infty$, which typically marks the threshold between a vanishing and a diverging asymptotic GGA potential. The AK13 functional implements this and additional requirements with the choice

$$
F^{\mathrm{AK} 13}(s)=1+B_{1} s \ln (1+s)+B_{2} s \ln [1+\ln (1+s)],
$$

where the constants $B_{1}=2 / 27+8 \pi / 15$ and $B_{2}=4 / 81-$ $8 \pi / 15$ have been determined in a nonempirical fashion. This asymptotic behavior has been adopted from the model potential of Becke and Johnson (BJ) [36], which proposes a semilocal correction to the Slater potential [57] utilizing the positively defined kinetic-energy density,

$$
\tau=\frac{1}{2} \sum_{i} f_{i}\left|\nabla \varphi_{i}\right|^{2}
$$

to mimic missing exchange features,

$$
v_{\mathrm{x}}^{\mathrm{BJ}}=v_{\mathrm{x}}^{\text {Slater }}+C_{\Delta v} \sqrt{\frac{2 \tau}{n}},
$$

where $C_{\Delta v}=\sqrt{5 / 12} / \pi$, and the occupation numbers $f_{i}$.
Concerning their limiting value, both potentials rely semilocally on the fact that far outside the system, the density as well as $\tau$ are governed by the highest occupied orbital. Under the assumption of spherical symmetry, this leads to the asymptotic relation [58]

$$
n(\mathbf{r}) \sim n_{0}|\mathbf{r}|^{q} \exp (-\alpha|\mathbf{r}|) \quad \text { as } \quad|\mathbf{r}| \rightarrow \infty,
$$

where $n_{0}$ and $q$ are system-dependent constants. The decay parameter

$$
\alpha=2 \sqrt{-2\left(\varepsilon_{\mathrm{ho}}-v_{\mathrm{x}}^{\infty}\right)}
$$

is determined by the highest occupied eigenvalue $\varepsilon_{\mathrm{ho}}$ relative to the limiting value of the potential $v_{\mathrm{x}}^{\infty}=\lim _{|\mathbf{r}| \rightarrow \infty} v_{\mathrm{x}}(\mathbf{r})$. Additional asymptotic relations involving the spatial derivatives of the density follow hereby:

$$
\begin{aligned}
\frac{u}{s^{3}} & \sim 1, \quad \frac{t}{s^{2}} \\
2 \gamma n^{1 / 3} s & \sim \alpha, \quad \frac{\tau}{n} \sim \frac{2}{3} \frac{1}{\ln (s)}, \\
& \sim \alpha,
\end{aligned}
$$

as $|\mathbf{r}| \rightarrow \infty$. Utilizing these, one can calculate the limits

$$
v_{\mathrm{x}}^{\mathrm{AK} 13, \infty}=-\frac{A_{\mathrm{x}} B_{1} \alpha}{6 \gamma}, \quad v_{\mathrm{x}}^{\mathrm{BJ}, \infty}=\frac{C_{\Delta v} \alpha}{2},
$$

to show the asymptotic similarities of both potentials. Thus, the limit of the AK13 potential approximately equates to $68 \%$ of the limit of the BJ potential. For further discussions, we note that the limit of the AK13 potential relies on the cancellation of the first-order terms $\propto s \ln (s)$, while the limit of the BJ potential is determined by a single first-order term.

Solving Eqs. (9) and (11) for a self-consistent value of $v_{\mathrm{x}}^{\infty}$ gives

$$
v_{\mathrm{x}}^{\infty}=Q\left(1+\sqrt{1-2 \varepsilon_{\mathrm{ho}} / Q}\right)
$$

with, respectively, $Q=\left(A_{\mathrm{x}} B_{1} / 6 \gamma\right)^{2}$ in the case of AK13 and $Q=\left(C_{\Delta v} / 2\right)^{2}$ in the case of BJ. Hence, the limiting values of both semilocal potentials depend on the value of the highest occupied eigenvalue and therefore change discontinuously if, e.g., an additional fraction of an electron is added to the system.

As the limiting value of the exact exchange-correlation potential equals zero [59], it is tempting [39,55] to apply a constant shift $v_{\mathrm{x}}^{\mathrm{DD}}$ to both semilocal potentials,

$$
v_{\mathrm{x}}^{0}(\mathbf{r})=v_{\mathrm{x}}^{\mathrm{SL}}(\mathbf{r})+v_{\mathrm{x}}^{\mathrm{DD}},
$$

where $v_{\mathrm{x}}^{\mathrm{DD}}=-\lim _{|\mathbf{r}| \rightarrow \infty} v_{\mathrm{x}}^{\mathrm{SL}}(\mathbf{r})=-v_{\mathrm{x}}^{\infty}$. Due to this realignment, the semilocal limiting value $v_{\mathrm{x}}^{\infty}$ gives rise to a nonlocal discontinuity of the potential triggered by a change in the value of the highest occupied eigenvalue. Thus, both realigned versions of the AK13 and the BJ potential mimic a feature associated with the derivative discontinuity (DD) [16] of the exact exchange (EXX) functional. Moreover, both models for exchange share additional attractive properties such as a step structure in the potential for well-separated subsystems and an improved shell structure in the potential for atoms [36,55]; in bulk systems, they show band gaps, band structures, and optical dielectric constants closer to EXX results $[50,55,60]-$ and are thus typically in better agreement with experiments. Hence, AK13 and BJ functionals implement several promising features. In terms of qualitative results, they are quite similar 
with the decisive advantage that the AK13 potential is an actual functional derivative while the BJ potential is not $[37,52,54]$.

However, as explained in Sec. I, we have now identified a number of anomalies and general difficulties caused by the construction scheme summarized above. Some stem from specific choices made in the construction of AK13 and could thus potentially be circumvented by improved design criteria. However, others appear more intimately coupled to the key feature of an asymptotically nonvanishing potential.

\section{AK13 FOR SYSTEMS WITH FRACTIONAL PARTICLE NUMBERS}

In this section, we will point out some features that AK13 shows when particle-number variations are explicitly considered. The shift $v_{\mathrm{x}}^{\mathrm{DD}}$ must be carefully examined in this context. For a system with fixed integer electron number, the constant shift of the potential by $v_{\mathrm{x}}^{\mathrm{DD}}$ serves two purposes: On the one hand, this shift realigns the zero of the eigenvalues, $\left\{\varepsilon_{i}^{0}=\varepsilon_{i}+v_{\mathrm{x}}^{\mathrm{DD}}\right\}$, onto the limiting value of the potential. This is a natural choice, as it separates bound from unbound states. One the other hand, the realignment of the potential introduces a nonlocal mechanism akin to the discontinuity of the exchange optimized effective potential (OEP) [61], a feature that is associated with the DD of the corresponding energy functional.

Such a shift is fully in line with the Hohenberg-Kohn theorem [1], as the effective potential is only determined up to an arbitrary constant. One may further argue that strictly speaking such a shift does not affect observables: It does not change the density, but only affects the Kohn-Sham eigenvalues, which are auxiliary quantities. A subtlety in this argument is related to the highest occupied eigenvalue, which equals minus the first ionization potential in exact DFT $[59,62]$ and therefore can be equated to an observable. One may thus wonder whether in a functional such as AK13 the shifted or the unshifted highest occupied eigenvalue should be used as an approximation to the first ionization potential. As the self-consistent density from such a functional decays according to Eq. (8), i.e., the asymptotic decay is governed by the shifted highest occupied eigenvalue, $\varepsilon_{\text {ho }}-v_{\mathrm{x}}^{\infty}$, whereas the decay of the exact density is likewise determined by the ionization potential, it seems reasonable to use the negativeshifted eigenvalue as an approximation to the ionization potential. In practice, the shifted AK13 eigenvalues generally are in better [55] agreement with the ionization potential from EXX (and thus also experimental values) than the highest occupied eigenvalues of other, commonly used, semilocal functionals [63].

However, the idea of shifting the eigenvalues can also be seen more critically when one adopts a different perspective. Consider the behavior of AK13 within the ensemble extension of DFT by Perdew et al. [16], i.e., the generalization to fractional particle numbers. In this framework, the absolute offset of the exchange-correlation potential is fixed and the exchange-correlation potential, $v_{\mathrm{xc}}=\delta E_{\mathrm{xc}} / \delta n$, is defined uniquely for a given energy functional $E_{\mathrm{xc}}$. This can be understood directly from Janak's theorem [65],

$$
\frac{\partial E}{\partial N}=\epsilon_{\mathrm{ho}}(N)
$$

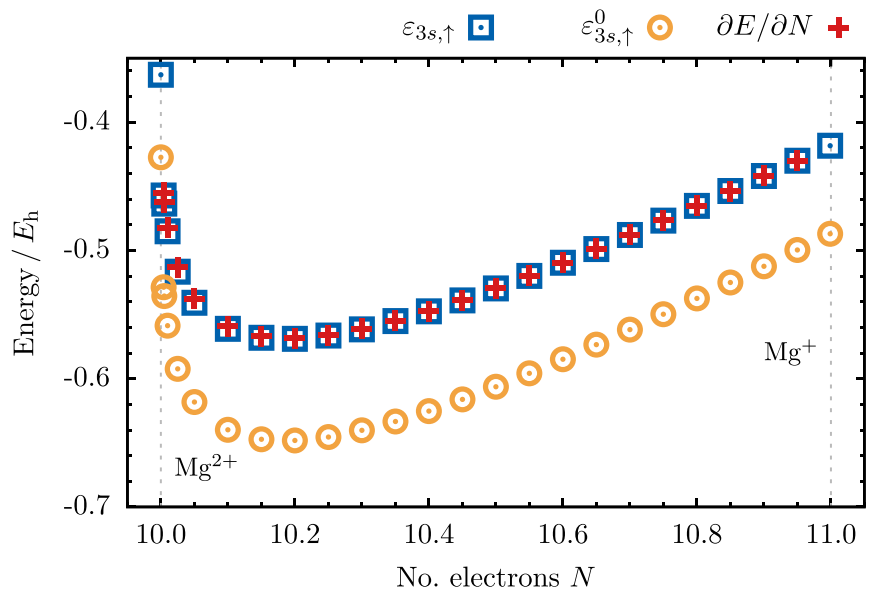

FIG. 1. Highest occupied orbital energy $\varepsilon_{3 s, \uparrow}$ corresponding to the semilocal AK13 potential [see Eq. (3)] $\varepsilon_{3 s, \uparrow}^{0}=\varepsilon_{3 s, \uparrow}+v_{\mathrm{x}}^{\mathrm{DD}}$ corresponding to its realigned version [see Eq. (13)] and the totalenergy derivative $\partial E / \partial N$ as a function of the number of electrons $N$ for ionized atomic magnesium. $\partial E / \partial N$ is calculated using central nonuniform first-order finite differences and the values of $E(N)$ at the shown points. The data points are based on self-consistent calculations with a code for atoms originating from Ref. [64].

which establishes a direct link between the particle-number dependence of the energy functional and the absolute offset of the eigenvalue energies in the KS system. Hence, one is not allowed to shift the energy scale of the KS system (which would shift the potential and eigenvalues) without also modifying the energy functional.

Janak's theorem can also be used to numerically verify the correct absolute offset. We demonstrate this in the following for AK13 (and in Fig. 6 of Appendix A for EXX). Figure 1 confirms that in a straightforward extension of AK13 to ensemble DFT [66], the appropriate exchange potential is not the zero-aligned one $v_{\mathrm{x}}^{0}$, but the unshifted, semilocal potential $v_{\mathrm{x}}^{\mathrm{SL}}$. This should not be surprising since $v_{\mathrm{x}}^{\mathrm{SL}}$ is the unmodified expression given by a straightforward functional derivative of the AK13 energy functional of Eq. (1).

A major conclusion from Janak's theorem is that the straightforward application of the AK13 energy functional in ensemble DFT gives a functional derivative that does not explicitly exhibit a discontinuity, i.e., a discontinuous shift of the potential at integer particle number. We illustrate the difference between the AK13 potential and the EXX potential with respect to the discontinuity in Appendix A using $\mathrm{Mg}^{2+}$ as an example. Despite lacking this absolute overall shift, fractional particle AK13 reproduces the step structure in its asymptotic behavior that is associated with the shift. For example, for a single ion, when the fractional occupancy goes through an integer, the asymptotic potential incorporates a step related to the atomic-shell structure that moves inwards as the fractional particle number increases, qualitatively mimicking a behavior seen in the EXX potential $[55,67]$.

The discussion above may suggest the idea of adding a term to the AK13 energy functional with the sole responsibility of generating a discontinuous shift. We have explored this idea, and in Appendix B we discuss why such an energy correction term is not straightforward to construct. 

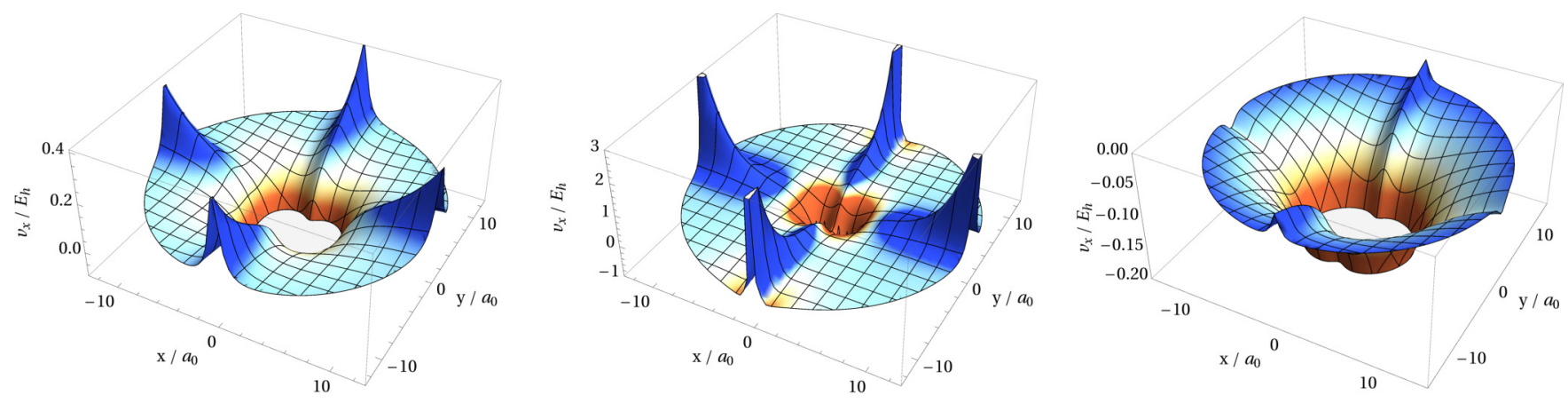

FIG. 2. Contour plot of the BJ, AK13, and EXX (OEP) potential landscapes of $\mathrm{O}_{2}$ for the majority spin channel in the plane containing the bond axis ( $x$ axis). The figure demonstrates that both semilocal potentials diverge exponentially along the nodal surfaces of the highest occupied orbital. Note that the scale of the potential axis as well as the coloring visualizing the height of the potentials differ. Due to serious numerical difficulties, which are intensified by the nodal surfaces [68], the BJ and AK13 potentials could not be calculated self-consistently and were instead evaluated on tightly converged self-consistent local spin density approximation (LSDA) orbitals. The calculations were performed with the all-electron code DARSEC [69], which is specialized on diatomic molecules and operates on a real-space grid of prolate-spheroidal coordinates.

Looking once more at the graph of Fig. 1 with the focus on the region close to $N \rightarrow 10^{+}$reveals that the AK13 energy response to a change in the fractional particle number deviates significantly from the exact behavior. Due to the critical behavior of $F^{\mathrm{AK} 13}(s)$ in the limit $s \rightarrow \infty$ [see Eq. (5)], the exchange-energy density of AK13 is highly sensitive to small changes of the electron density which alter its exponential decay. Addition of a fraction of an electron to the system by fractionally occupying a new orbital is such a change. The result is a short interval with high curvature of the $E(N)$ curve which deviates from the desired piecewise-linear behavior [16]. Thus, whereas the exact DD goes along with piecewise linearity, its semilocal imitation here is acting contrarily.

Finally, it is worthwhile to return to the discussion of whether it is more appropriate to use the shifted or the unshifted highest occupied eigenvalue to approximate the negative first ionization potential. At the beginning of this section, we had presented arguments for using the shifted eigenvalue. However, Janak's theorem shows that the highest occupied eigenvalue equals the total-energy difference between the $N-1$ and $N$ particle system for functionals that sufficiently fulfill the piecewise-linearity condition [16] (which AK13 does not). This applies regardless of whether or not the functional has a nonvanishing asymptotic potential. Therefore, from the perspective of Janak's theorem, one comes to the conclusion that from a formal standpoint, it is appropriate to identify the unshifted highest occupied eigenvalue with the negativeionization potential. Although this seems like a contradiction to the arguments given above, there is no formal mistake. These two different perspectives are possible due to the approximative nature of the functionals under consideration-the exact functional does not exhibit a nonvanishing asymptotic constant and is piecewise linear. From a pragmatic point of view, it makes sense to adopt the perspective which gives better results in practice, i.e., for AK13 to use the shifted eigenvalue.

\section{ENERGIES AND ENERGETICS}

AK13 can be seen as an improvement over the BJ model especially because it has an energy functional. However, as discussed in past works that go back to the original AK13 paper, the accuracy of total energies from this functional is not as good $[40,45,55,70]$ as from established GGAs, e.g., the one of Perdew, Burke, and Ernzerhof (PBE) [71]. Instead, one finds that the energetics displayed upon structural relaxation are distorted beyond what seems reasonable even for an exchange-only functional (see Ref. [72] and the Supplemental Material of Ref. [55]). This was the topic of a recent work [72], with a typical example of a bad structural relaxation being AlAs, which deviates from the experimental lattice constant by $16 \%$. Another similar indication of something missing from the AK13 total energies is the self-consistent field (SCF) results for atomic ionization; AK13 SCF energies deviate more from exact-exchange results than those of the local density approximation (LDA) [55].

\section{DIVERGENT POTENTIAL ALONG NODAL SURFACES}

In many finite systems, the highest occupied ground-state $\mathrm{KS}$ orbital has a nodal surface extending to infinity. The asymptotic density is normally governed by the highest occupied orbital; however, this is not necessarily the case for all its asymptotic properties in the vicinity of nodal surfaces. We recently pointed out that this region is troublesome for many semilocal exchange functionals [68]. In summary, the behavior of EXX on nodal surfaces is a protruding ridge along such regions [25-28]. Ordinary semilocal potentials such as the LDA potential decay rapidly in the asymptotic region in a way that mostly does not distinguish nodal surfaces. Energy functionals with divergent enhancement factors can display a range of different behaviors, but, if the divergence is strong enough, the potential will diverge exponentially along the nodal surface. Examples of such functionals are the BJ potential, the Becke 1988 exchange functional [73], and AK13. Of these, AK13 displays the strongest divergence; it is twice as strongly diverging as the BJ model. A demonstration of this issue is presented in Fig. 2, which features the divergent $\mathrm{BJ}$ and AK13 potentials in comparison with the EXX (OEP) potential evaluated for $\mathrm{O}_{2}$, a system with nodal surfaces of the highest occupied KS orbital. As previously discussed 
[68], these divergences are not only theoretically worrisome but also lead to major numerical difficulties when trying to converge calculations for finite systems with nodal surfaces. The $\mathrm{O}_{2}$ molecule that we show here presents a situation that somewhat differs from the common cases discussed previously in Ref. [68], as here the doubly degenerate highest occupied orbitals of the majority spin channel form a nodal line along the bond axis in addition to a nodal plane between the two oxygen nuclei, and also the two degenerate orbitals below the highest occupied ones exhibit this nodal line along the bond axis. As a consequence of this unusual electronic structure, the density of the majority spin channel along the bond axis is asymptotically dominated by the fifth-highest occupied orbital [74] and produces a rare feature in the asymptotic exact exchange potential as well: In addition to a ridge that results from a positive limiting value along the nodal plane, a furrow related to an uncommon negative limiting value shows up in the potential along the nodal line of the bond axis, as can be seen in Fig. 2(c).

\section{SECOND-ORDER ASYMPTOTICS}

Next we discuss an unintended behavior of the AK13 construct that relates to its asymptotic second-order term, $\sim B_{2} s \ln [\ln (s)]$ as $s \rightarrow \infty$, in the enhancement factor $F^{\mathrm{AK} 13}(s)$; cf. Eq. (5). The original motivation [55] of the $s \ln [\ln (s)]$ term was to mimic the leading asymptotic behavior $v_{\mathrm{x}} \sim-c / z$ outside the surface of a half-infinite bulk system with $c$ a system-dependent prefactor and $z$ the distance to the surface. However, in leading asymptotic order, a term $\sim_{s} \ln [\ln (s)]$ results in a system-independent contribution to the potential $\propto 1 / z$. Nonetheless, this term is important as it balances the divergence of the enhancement factor in the limit $s \rightarrow \infty$ having the opposite sign of the leading term, $\sim B_{1} s \ln (s)$. This balance is needed to provide reasonable energies as well as to improve numerical evaluability of the potential in the asymptotic region of finite systems.

The drawback of this $B_{2}$ term becomes evident when evaluating the asymptotics of the AK13 potential in detail,

$$
\begin{aligned}
v_{\mathrm{x}}^{\mathrm{AK} 13}(r) \sim & -\frac{A_{\mathrm{x}} B_{1}}{6 \gamma} \alpha+\frac{A_{\mathrm{x}} B_{2}}{\gamma} \frac{\ln (\alpha r / 3)}{r} \\
& +\frac{A_{\mathrm{x}}}{\gamma}\left[\left(B_{1}-\frac{3}{2} B_{2}\right)-B_{1} \ln \left(2 \gamma n_{0}^{1 / 3} / \alpha\right)\right] \frac{1}{r},
\end{aligned}
$$

as $r=|\mathbf{r}| \rightarrow \infty$ and given the asymptotic density of Eq. (8) with $q=0$ for simplicity. The first term of Eq. (15) represents the positive system-dependent asymptotic constant of AK13, whereas the second and third terms describe how the potential approaches this nonvanishing asymptotic constant. The system-independent contribution to the third term gives by construction the desired $-1 / r$ behavior. However, this term is asymptotically dominated by the second term $\propto \ln (r) / r$, which has a positive sign. Therefore, the asymptotic constant of the AK13 potential is ultimately approached from above and the potential has a local maximum in the asymptotic region. The latter is approached too fast for the potential to be able to bind additional electrons [70].

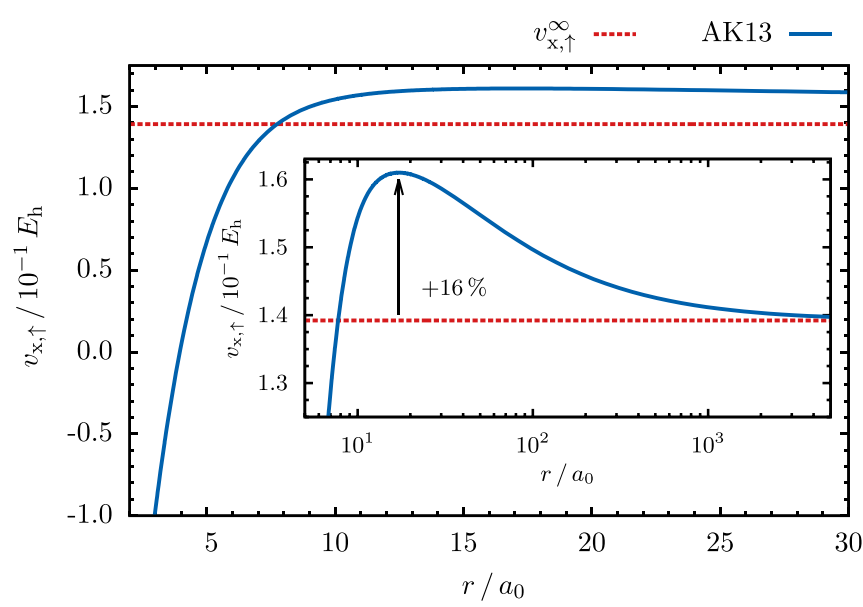

FIG. 3. AK13 potential evaluated for the exact hydrogen groundstate density. Looking at the outer graph, i.e., a typical computational length scale, the potential seems to approach a positive constant unequal to $v_{\mathrm{x}}^{\infty}$ given by Eq. (11). The inset shows the same potential on a logarithmic scale.

A second consequence is exemplified by Fig. 3. It shows the AK13 potential for the exact hydrogen ground-state density. Within the typical length scale of a numerical electronic structure calculation of less than 30 Bohr radii, the AK13 potential seemingly approaches an asymptotic constant which is $16 \%$ higher than the actual limiting value given by Eq. (11). The true limiting value is approached only within a length scale of several-thousand Bohr radii. This is a consequence of $\ln (r) / r$ decaying only marginally more slowly than $1 / r$. This undesirable behavior can be noticed in numerical calculations of other systems as well. The theoretical limiting value of the potential is therefore of only limited significance in typical calculations.

This drawback could be corrected by modifying the construction of AK13. In such a revised construction of AK13, one could, e.g., replace the $B_{2} s \ln [1+\ln (1+s)]$ term by a term that exhibits an asymptotic behavior $\propto s$ as $s \rightarrow \infty$ and maintains reasonable balance with the original $B_{1}$ term.

\section{EXTERNAL ELECTRICAL FIELDS}

The hope for improved charge-transfer characteristics spurred some of the investigations of the BJ potential [39,41,52], and corresponding hopes may have been associated with AK13. Mimicking the field-counteracting behavior of exact exchange [31,75] with the semilocal BJ potential, however, turned out to be difficult. In order to clarify the situation for AK13, here we look at a standard test case. We study external electrical fields that are weak and linear, i.e., their contribution to the Hamiltonian is $\mathcal{F}_{z}$ with some small field amplitude $\mathcal{F}$ and the $z$ axis chosen in the direction of the field. Such "infinitely large, weak fields" are, e.g., used to calculate the electrical response of molecules [76], in particular of molecular chains, within DFT. In the following, we study a frequently used [31,75-87] model molecular system: the hydrogen chains.

Given the asymptotic similarities of the AK13 and BJ potential pointed out in Sec. II, it does not come as a surprise 


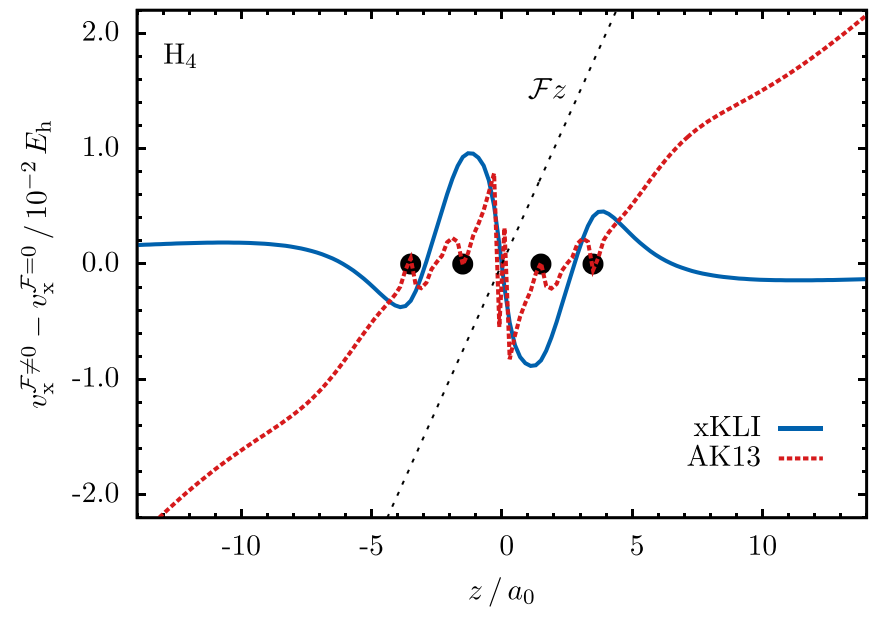

FIG. 4. Difference between the exchange potential of calculations with and without an external electric field of strength $\mathcal{F}=5 \times$ $10^{-3} E_{h} / a_{0}$ for a $\mathrm{H}_{4}$ chain (atom positions are indicated by circles). The solid blue line shows the desired field-counteracting behavior of EXX within the Krieger-Li-Iafrate (KLI) approximation (xKLI). AK13 (dashed red line) is tilted in the direction of the field, similarly to BJ (see Fig. 5 of Ref. [39]). The AK13 potentials are not calculated self-consistently due to the discussed numerical difficulties, but evaluated on self-consistent xKLI KS orbitals in PARSEC [88] with Giannozzi pseudopotentials [89], ellipsoidic boundaries with semiaxis of $10 a_{0}$ perpendicular to and $30 a_{0}$ along the chain, and a grid spacing of $0.2 a_{0}$.

that in the presence of an external electric field, AK13 shows the same surprising unphysical behavior as BJ [39], i.e., the potential is asymptotically tilted in the direction of the external field and does not go to zero. Figure 4 demonstrates this by showing the difference between the exchange potential of a chain of four hydrogen atoms in a weak electric field and the potential with no external field, $v_{\mathrm{x}}^{\mathcal{F} \neq 0}-v_{\mathrm{x}}^{\mathcal{F}=0}$. The approximately linear tilt of the AK13 potential in the asymptotic region arises solely in the presence of the external field. It can be understood as a consequence of the deviation of the asymptotic density from Eq. (8) in response to the electric field. Terms of the AK13 potential that contribute to an asymptotic constant when Eq. (8) holds, i.e., terms that arise from $F(s) \sim B_{1} s \ln (s)$ as $s \rightarrow \infty$, now yield (in first order) a linearly diverging contribution to the potential.

The leading asymptotic behavior in the presence of a linear electric field can be retraced by utilizing the one-dimensional Airy gas model in the spirit of the analogous calculation for the BJ potential of Ref. [39]. While the result of the corresponding AK13 calculation for this one-dimensional model is in full analogy to the BJ result [90], it is not quantitatively transferable to the three-dimensional case, as the asymptotics of the AK13 potential show a direct dependence on the spatial dimension [55] (which BJ does not show). The one-dimensional result and Fig. 4 suggest that

$$
v_{\mathrm{x}}^{\mathrm{AK} 13}(\rho=0, z) \sim v_{\mathrm{x}}^{\mathrm{AK} 13, \infty}+C \frac{\mathcal{F} z}{\sqrt{-2 \epsilon_{\mathrm{ho}}}}
$$

in the asymptotic region while $|z| \ll\left|\mathcal{F} / \epsilon_{\text {ho }}\right|$ with some constant $C>0$ and $\rho=\sqrt{x^{2}+y^{2}}$.
In Ref. [39], the relation that is the analog of Eq. (16) for BJ was used to justify a linear, field-dependent correction to the BJ potential. Since this correction is applied globally, it fixes the unphysical tilt in the asymptotic region, but more importantly leads to a slope counteracting the external field in the inner region, thus mimicking response physics that could previously not be captured by semilocal constructions. The resulting total $\mathrm{BJ}$ potential response to the electrical field is remarkably close to exact-exchange calculations and yields vastly improved polarizabilities [39,52]. Consequently, an analogue correction to AK13 suggests itself. However, the one important benefit of $\mathrm{AK} 13$ over $\mathrm{BJ}$ is the fact that its potential is an actual functional derivative of a well-defined energy expression. Thus, to maintain a correspondence between energy and potential, the field-dependent correction to AK13 has to originate from an energy correction. Given that this energy correction cannot explicitly depend on the external electrical field in order to yield consistent polarizabilities from energy and potential [76], there is no clear way to create such a term.

\section{INFLUENCE OF THE NUMERICAL REPRESENTATION}

Next we will discuss how strongly the chosen numerical representation (basis set) of the KS orbitals influences calculations of finite systems for functionals with asymptotically nonvanishing potentials. This can hardly be referenced as anomalies in the functionals themselves, but is still an issue of high relevance for their application. For usual (semi)local functionals, the accuracy of the numerical representation of the far asymptotic density is typically of little concern because the energy and potential of such functionals are not sensitive to these regions of space. The AK13 energy and the AK13 and BJ potentials, however, are by construction highly sensitive to the precise decay of the density, which is measured by ratios such as $|\nabla n| / n$. When this ratio is numerically evaluated, a representation of the asymptotic density that is not highly accurate can cause serious numerical problems, e.g., instabilities. Small numerical errors might, for example, prevent the required cancellation of the leading-order terms $\propto s \ln (s)$ in the AK13 potential, and therefore cause a linearly growing error in the potential in the asymptotic region. This then can amplify the numerical error in the next step of a KS iteration.

In the following, we discuss three common approaches to represent KS orbitals, and their implications for the asymptotic potential: real-space grids [69,88,91-94], Slater-type orbitals (STOs) [95], and Gaussian-type orbitals (GTOs) [96,97].

The representation of orbitals on real-space grids is the most flexible, yet the computationally most expensive one of these three. The relative numerical accuracy of this representation is typically decaying in the far asymptotic region, especially when looking at spatial derivatives represented via finite differences. The restriction to a necessarily finite grid also introduces boundary effects. As a consequence, the abovediscussed problems of evaluating the AK13 potential in the asymptotic region are strongly noticeable and make threedimensional, grid-based AK13 calculations extremely hard.

The usage of either STOs or GTOs circumvents some of these problems. The reason is that, on the one hand, 
critical ingredients to the semilocal potential, i.e., the density derivatives $|\nabla n|$ and $\nabla^{2} n$, are analytically accessible and are thereby numerically arbitrarily accurate, even in the asymptotic region. On the other hand, both representations require only a relative small basis-set size, as these sets are highly tailored and specific to each atom. Therefore, the degrees of freedom and, consequently, the susceptibility to instabilities are considerably reduced compared to real-space methods. Yet, there is a non-negligible price to pay for this "convenience": It is the qualitatively wrong behavior of the density in the asymptotic region that occurs for both STOs and GTOs. It in turn has the following conceptional implications: In the case of STOs, the issue is that the exponential decay of the KS orbitals is predefined by the basis set. This means that when using semilocal density functionals with an asymptotically nonvanishing potential, the nonzero limiting value of the potential, $v_{\mathrm{x}}^{\infty}$, is not determined by the system within the self-consistent calculation, but rather is given by the basis set and thus already fixed prior to the actual calculation. Therefore, in the case of STOs, neither Eq. (9) nor Eq. (12) hold strictly. Yet, they can hold approximately when a reasonable basis set of STOs is chosen.

The issue with GTOs is similar, but even more apparent. GTOs sacrifice the correct orbital asymptotic that is achieved with STOs. Thus, in the case of GTOs, the asymptotic relation $n(r) \propto \exp \left(-\beta r^{2}\right)$ as $r \rightarrow \infty$ replaces Eq. (8), which results in a qualitatively different behavior of semilocally nonvanishing potentials: Instead of approaching a systemdependent asymptotic constant outside of a finite system, the AK13 and BJ potentials spuriously diverge linearly to positive infinity, e.g.,

$$
v_{\mathrm{x}}^{\mathrm{AK} 13}(r) \sim-\frac{2 A_{\mathrm{x}} B_{1}}{3 \gamma} \beta r,
$$

as $r \rightarrow \infty$. Additionally, the asymptotic slope of these potentials is akin to the asymptotic constant in the case of STOs, predefined by the basis set via the value of $\beta$.

Hence, one should be aware that semilocal density functionals with an asymptotically nonvanishing potential show significantly higher demands on the numerical representation of the KS orbitals, especially in the asymptotic region. To summarize, real-space methods provide, in principle, the qualitatively most accurate representation in this region, but are susceptible to instabilities, whereas STOs or GTOs provide higher stability, but imply a qualitatively wrong asymptotic density and potential.

\section{SUMMARY AND CONCLUSIONS}

In this paper, we have investigated a set of anomalous features of semilocal functionals with nonvanishing asymptotic exchange potentials, with a particular focus on AK13 and its predecessor BJ. We also commented on the numerical difficulties that appear when evaluating such functionals in standard electronic structure codes. In particular, we have discussed misfeatures seen in the direct application of AK13 in ensemble DFT for systems with fractional particle numbers, inaccurate energies and energetics, divergent potentials along nodal surfaces, nonphysical response to an external electric dipole field, and practical difficulties due to the numerical orbital representation used. The issues we have identified and discussed in this work provide a formidable challenge for the future development of functionals with nonvanishing potentials.

There are different approaches one can try to overcome these difficulties and move forward with the aim to incorporate important exact-exchange features into functionals with modest computational cost. One option is to continue the development of semilocal density functionals with asymptotically nonvanishing potentials. Extending the AK13 construction idea, one can try to explicitly tackle each deficit that we have pointed out here. This is a major challenge, but when carried out successfully such a strategy should lead to a formally satisfying consistent energy-potential pair. A second option is to follow the idea of GLLB $[46,47]$ and construct model potentials that incorporate some of the desired exact-exchange features via an explicit orbital and eigenvalue dependence. This type of scheme can provide numerically robust potentials that do not suffer from the issues that are related to the semilocal realization of a nonvanishing asymptotic constant. A downside of this approach is that such constructions are not functional derivatives of a corresponding energy functional. This implies serious drawbacks that we have already discussed briefly in Sec. I, such as instabilities in time-dependent DFT and no possibility for geometry optimization. A third option is meta-generalized-gradient approximations (meta-GGAs), i.e., energy functionals that are semilocal not in the density but in the orbitals and make use of, e.g., the kinetic-energy density $\tau$. As discussed previously by Eich and Hellgren [98], meta-GGAs used in the Kohn-Sham scheme in general have a derivative discontinuity due the nonlocal character of their multiplicative potential. However, as commonly used metaGGAs to date largely underestimate the exchange derivative discontinuity and related properties, it remains to be seen whether the desired potential features can be captured on the meta-GGA level to an extent that is useful in practice.

\section{ACKNOWLEDGMENTS}

S.K. and T.A. acknowledge financial support by the German-Israeli Foundation for Scientific Research and Development. T.A. acknowledges support by the University of Bayreuth Graduate School. R.A. acknowledges financial support from the Swedish Research Council (V.R.), Grant No. 2016-04810, and the Swedish e-Science Research Centre (SeRC).

\section{APPENDIX A: COMPARISON OF AK13 AND EXX POTENTIALS FOR FRACTIONAL PARTICLES NUMBERS}

Section III discussed the straightforward extension of AK13 to ensemble DFT using fractional particle numbers. We argued on general grounds, and explicitly demonstrated for ionized atomic magnesium in Fig. 1, that the AK13 (ensemble) potential does not exhibit a global discontinuous shift at integer particle number. Yet, the AK13 potential reproduces a step in the asymptotic region that is typically associated with such a discontinuous shift. In order to further discuss and illustrate the relation between the step structure and the shift, we compare the functional derivative with respect to the density of the EXX 


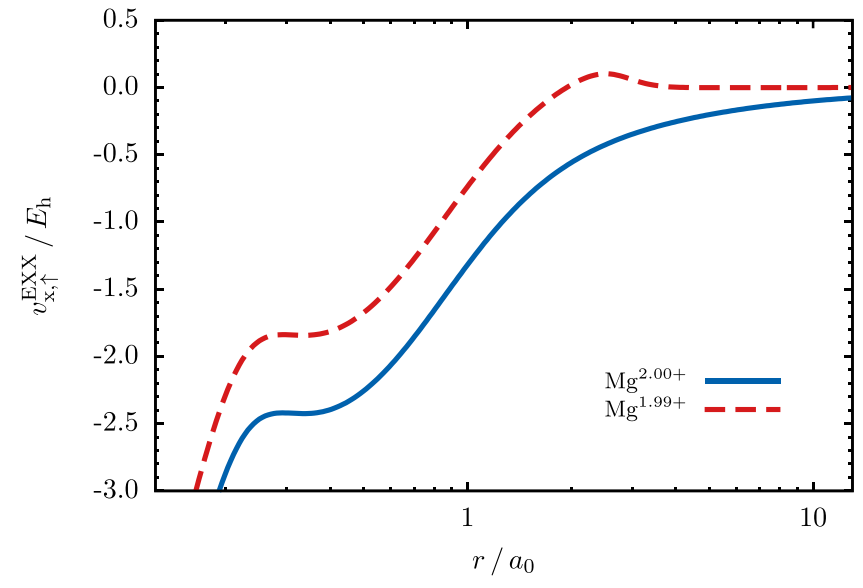

(a) $\operatorname{EXX}$

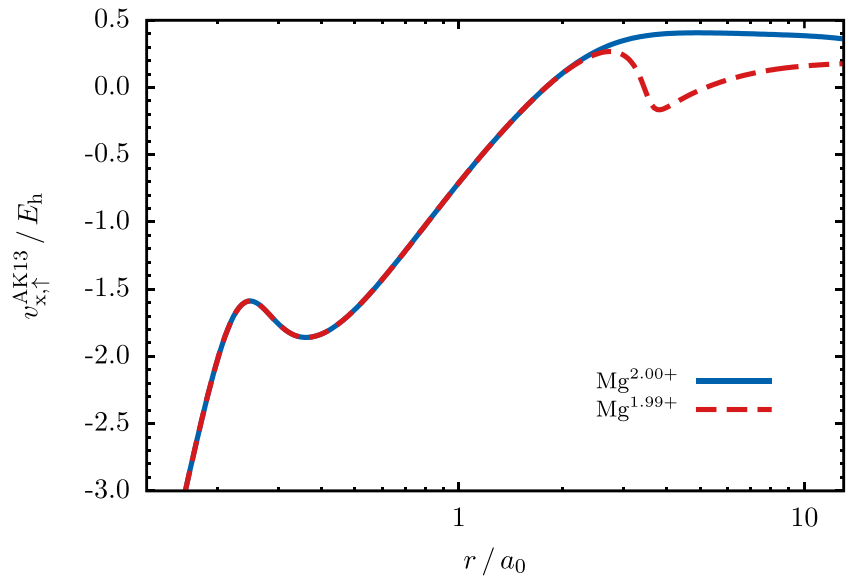

(b) AK13

FIG. 5. Change of the EXX (in the KLI approximation) and the (unshifted) AK13 spin-up potentials upon addition of a fraction of an electron to double-ionized atomic magnesium. The potentials are based on self-consistent calculations with a code for atoms operating on a logarithmic grid and originating from Ref. [64]. While the EXX potential jumps globally and discontinuously at integer particle numbers, solely the asymptotic limiting value of the AK13 potentials jumps. Similar plots were shown for BJ in Fig. 2 of Ref. [39] and for AK13 in Fig. 4 of Ref. [55].

energy, on the one hand, to the unshifted functional derivative of the AK13 energy functional for $\mathrm{Mg}^{2+}$, on the other hand, as we add a small fraction of an electron to the system. The functional derivatives are shown in Fig. 5 for a fractional occupation of one percent, i.e., $N=10+\epsilon$ with $\epsilon=0.01$. By virtue of the spin-scaling relation for exchange [99], it is to be expected that only the exchange potential of the spin channel in which the particle number changes is substantially affected and can show a discontinuity or step. This is also reflected in the AK13 functional and becomes manifest in the fact that the asymptotic constant of the AK13 potential is spin dependent and may differ between spin channels. Therefore, we deliberately focus our discussion only on this spin channel, which is here chosen to be the up-spin channel.

Upon addition of a fraction of an electron, the functional derivative of the EXX energy jumps up in the interior region, $r \lesssim 2 a_{0}$, by approximately a constant, $\Delta_{\mathrm{X}}^{\mathrm{EXX}}$, whereas in the asymptotic region the EXX potential maintains the same limiting value as the EXX potential at integer number of electrons,

$$
\lim _{r \rightarrow \infty} v_{\mathrm{x}, \uparrow}^{\mathrm{EXX}, N=10+\epsilon}(r)=\lim _{r \rightarrow \infty} v_{\mathrm{x}, \uparrow}^{\mathrm{EXX}, N=10}(r)=0 .
$$

As $\epsilon \rightarrow 0^{+}$, the step between these two regions moves outwards and the discontinuous shift of the potential at integer particle number becomes apparent; the potentials at any point $r$ at a finite distance differ by just a constant shift in this case,

$$
\lim _{\epsilon \rightarrow 0^{+}} v_{\mathrm{x}, \uparrow}^{\mathrm{EXX}, N=10+\epsilon}(r)-v_{\mathrm{x}, \uparrow}^{\mathrm{EXX}, N=10}(r)=\Delta_{\mathrm{x}}^{\mathrm{EXX}} .
$$

In the case of the unshifted AK13 functional derivative, the situation is different: In the interior region, the potentials with and without fractional particle number overlap perfectly due to their semilocal nature. However, by construction, the limiting value of the AK13 potential decreases discontinuously upon addition of a fraction of an electron [cf. Eq. (12)], thus

$$
\lim _{r \rightarrow \infty}\left[v_{\mathrm{x}, \uparrow}^{\mathrm{AK} 13, N=10+\epsilon}(r)-v_{\mathrm{x}, \uparrow}^{\mathrm{AK} 13, N=10}(r)\right]=\Delta_{\mathrm{x}}^{\mathrm{AK} 13} .
$$

The result is a step downward in the fractional AK13 potential between the interior and the asymptotic region. Similar to the step that is present in the EXX potential, the step between these two regions moves outwards as $\epsilon \rightarrow 0^{+}$. However, as the AK13 potentials with different particle numbers differ in the asymptotic region and not in the interior region, there remains no global discontinuous shift of the potential,

$$
\lim _{\epsilon \rightarrow 0^{+}} v_{\mathrm{x}, \uparrow}^{\mathrm{AK} 13, \mathrm{~N}=10+\epsilon}(r)-v_{\mathrm{x}, \uparrow}^{\mathrm{AK} 13, N=10}(r)=0,
$$

in contrast to EXX.

This contrast can also be summarized in the following order of limits relation, which in the case of the EXX potential (as well as the exact $\mathrm{xc}$ potential) reads

$$
\begin{aligned}
& \lim _{\epsilon \rightarrow 0^{+}} \lim _{|\mathbf{r}| \rightarrow \infty}\left[v_{\mathrm{x}}^{N_{0}+\epsilon}(\mathbf{r})-v_{\mathrm{x}}^{N_{0}-\epsilon}(\mathbf{r})\right]=0, \\
& \lim _{|\mathbf{r}| \rightarrow \infty} \lim _{\epsilon \rightarrow 0^{+}}\left[v_{\mathrm{x}}^{N_{0}+\epsilon}(\mathbf{r})-v_{\mathrm{x}}^{N_{0}-\epsilon}(\mathbf{r})\right]=\Delta_{x},
\end{aligned}
$$

whereas the relation is reversed for $\mathrm{AK} 13$,

$$
\begin{array}{r}
\lim _{\epsilon \rightarrow 0^{+}} \lim _{|\mathbf{r}| \rightarrow \infty}\left[v_{\mathrm{x}}^{N_{0}+\epsilon}(\mathbf{r})-v_{\mathrm{x}}^{N_{0}-\epsilon}(\mathbf{r})\right]=\Delta_{x}^{\mathrm{AK} 13}, \\
\lim _{|\mathbf{r}| \rightarrow \infty} \lim _{\epsilon \rightarrow 0^{+}}\left[v_{\mathrm{x}}^{N_{0}+\epsilon}(\mathbf{r})-v_{\mathrm{x}}^{N_{0}-\epsilon}(\mathbf{r})\right]=0 .
\end{array}
$$

In the left-hand sides of these equations, we dropped the superscripts EXX and AK13 for brevity of notation. Thus, the qualitative difference between AK13 and EXX essentially comes down to a missing global shift of the AK13 functional derivative. This missing shift is precisely the one proposed to be added in relation to Eq. (13) and discussed in Sec. III as being in line with the Hohenberg-Kohn theorem for integer particle systems but inadmissible in ensemble DFT.

If one is familiar with the OEP (or KLI) construction in detail, one may wonder why we chose to align the EXX potential for $N=10+\epsilon$ such that it goes to zero at infinityafter all, one has to make a deliberate choice for the asymptotic constant in the OEP construction [61,67]. So if we chose to 


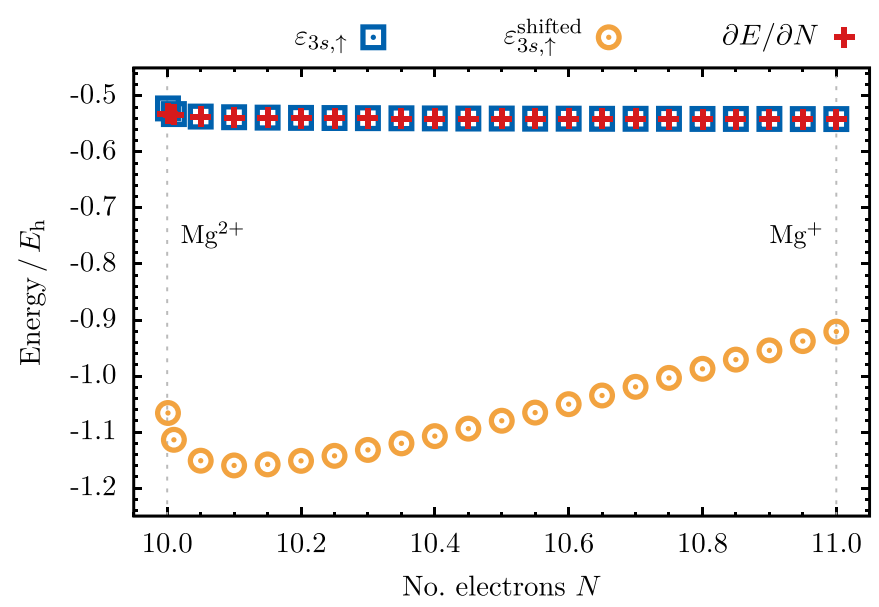

FIG. 6. Highest occupied orbital energy $\varepsilon_{3 s, \uparrow}$ corresponding to the EXX (KLI) potential aligned to zero at infinity, $\varepsilon_{3 s, \uparrow}^{\text {shifted }}$ corresponding to the EXX (KLI) potential aligned in the interior region with the potential at integer number of electrons $\left(\mathrm{Mg}^{2+}\right)$, and the total-energy derivative $\partial E / \partial N$ as a function of the number of electrons $N$ for ionized atomic magnesium. $\partial E / \partial N$ is calculated using central nonuniform first-order finite differences and the values of $E(N)$ at the shown points. The data points are based on self-consistent calculations with the all-electron code DARSEC [69].

not align the AK13 potential at zero, why did we choose to align the EXX potential? The answer to this question is that as mentioned previously, we have no liberty in choosing the constant in ensemble DFT but have to accept the constant that comes out of the functional derivative. Figure 6 demonstrates via Janak's theorem that the EXX potential aligned to zero at infinity corresponds to the functional derivative of the EXX energy, whereas Fig. 1 shows that the unaligned AK13 potential is the functional derivative of the AK13 energy. Therefore, Fig. 5 depicts the proper functional derivatives. We note in passing that for model potentials such as BJ or GLLB, the question of "properly aligning" is irrelevant, as these potentials are not functional derivatives to begin with.

\section{APPENDIX B: NONEXISTENCE OF A STRAIGHTFORWARD ENERGY CORRECTION}

As discussed in Sec. III, one might wonder if there exists a straightforward energy correction to the semilocal AK13 functional that realigns the potential and introduces a discontinuous shift of the potential in ensemble DFT. If such a correction could be devised, the corrected AK13 functional would take the form

$$
E_{\mathrm{x}}^{\mathrm{AK} 13,0}[n]=E_{\mathrm{x}}^{\mathrm{AK} 13}[n]+E_{\mathrm{x}}^{\mathrm{DD}}[n],
$$

where the functional derivative equals the realigned AK13 potential, as given by Eq. (13). We will in the following prove that the behaviors under uniform density coordinate scaling of Eqs. (13) and (B1) are in contradiction. This disproves the existence of a "straightforward" energy correction whose action is only a simple system-independent realignment of the potential to zero by a constant homogeneous shift in the whole system. We specifically note that therefore the following proof is valid only for shifts that are rigorously constant everywhere, i.e., including the boundary of the space that is considered.

Assume the functional $E_{\mathrm{x}}^{\mathrm{DD}}[n]$ exists. On addition to $E_{\mathrm{x}}^{\mathrm{AK} 13}[n]$, the combined exchange potential then is

$$
\frac{\delta E_{\mathrm{x}}^{\mathrm{AK} 13}[n]}{\delta n(\mathbf{r})}+\frac{\delta E_{\mathrm{x}}^{\mathrm{DD}}[n]}{\delta n(\mathbf{r})}=v_{\mathrm{x}}^{\mathrm{AK} 13}([n] ; \mathbf{r})+v_{\mathrm{x}}^{\mathrm{DD}}[n],
$$

where $v_{\mathrm{x}}^{\mathrm{DD}}[n]=-\lim _{|\mathbf{r}| \rightarrow \infty} v_{\mathrm{x}}^{\mathrm{AK} 13}(\mathbf{r})$ by assumption. Now, consider some well-behaved spherical-symmetric density $n(\mathbf{r})$ of a finite system, which satisfies the asymptotic relation of Eq. (8). Hence, by virtue of Eq. (9),

$$
v_{\mathrm{x}}^{\mathrm{DD}}[n]=-\lim _{|\mathbf{r}| \rightarrow \infty} v_{\mathrm{x}}^{\mathrm{AK} 13}([n] ; \mathbf{r})=-\frac{A_{\mathrm{x}} B_{1} \alpha}{6 \gamma} .
$$

Given this density, we define the uniform density path,

$$
n_{\lambda}(\mathbf{r})=\lambda^{3} n(\lambda \mathbf{r}) \quad \text { for } \quad \lambda \in(0 ; 1],
$$

and investigate the derivative of $E_{\mathrm{x}}^{\mathrm{DD}}[n]$ with respect to $\lambda$ along this path,

$$
\frac{d E_{\mathrm{x}}^{\mathrm{DD}}\left[n_{\lambda}\right]}{d \lambda}=\int \frac{\delta E_{\mathrm{x}}^{\mathrm{DD}}\left[n_{\lambda}\right]}{\delta n_{\lambda}(\mathbf{r})} \frac{d n_{\lambda}(\mathbf{r})}{d \lambda} d^{3} r,
$$

where $\delta E_{\mathrm{x}}^{\mathrm{DD}}\left[n_{\lambda}\right] / \delta n_{\lambda}(\mathbf{r})=v_{\mathrm{x}}^{\mathrm{DD}}\left(\left[n_{\lambda}\right] ; \mathbf{r}\right)$ and

$$
\frac{d n_{\lambda}(\mathbf{r})}{d \lambda}=3 \lambda^{2} n(\lambda \mathbf{r})+\lambda^{3} \mathbf{r} \cdot \frac{\partial n(\lambda \mathbf{r})}{\partial(\lambda \mathbf{r})} .
$$

As by construction $v_{\mathrm{x}}^{\mathrm{DD}}[n]$ has to system independently cancel the nonzero asymptotic value of the AK13 potential, one can show

$$
v_{\mathrm{x}}^{\mathrm{DD}}\left[n_{\lambda}\right]=-\frac{A_{\mathrm{x}} B_{1} \alpha \lambda}{6 \gamma}=\lambda v_{\mathrm{x}}^{\mathrm{DD}}[n]
$$

by evaluating $v_{\mathrm{x}}^{\mathrm{AK} 13}\left(\left[n_{\lambda}\right] ; \mathbf{r}\right)$ along the density path of Eq. (B4) and by applying Eq. (B3), respectively. Inserting this result together with Eq. (B6) into Eq. (B5) while applying the substitution $\lambda \mathbf{r} \rightarrow \mathbf{r}$ gives

$$
\begin{aligned}
\frac{d E_{\mathrm{x}}^{\mathrm{DD}}\left[n_{\lambda}\right]}{d \lambda} & =\int v_{\mathrm{x}}^{\mathrm{DD}}[n][3 n(\mathbf{r})+\mathbf{r} \cdot \nabla n(\mathbf{r})] d^{3} r \\
& =v_{\mathrm{x}}^{\mathrm{DD}}[n] \int \nabla \cdot[\mathbf{r} n(\mathbf{r})] d^{3} r=0,
\end{aligned}
$$

where we have utilized the divergence theorem in the final step with no boundary contribution due to $n(\mathbf{r})$ satisfying Eq. (8). Thus, the energy correction $E_{\mathrm{x}}^{\mathrm{DD}}[n]$ is invariant under uniform density scaling for a density that satisfies the asymptotics relations of Eq. (8),

$$
E_{\mathrm{x}}^{\mathrm{DD}}\left[n_{\lambda}\right]=E_{\mathrm{x}}^{\mathrm{DD}}[n] .
$$

Taking the functional derivative of Eq. (B9) with respect to $n(\mathbf{r})$ and applying the chain rule on the left-hand side then yields

$$
v_{\mathrm{x}}^{\mathrm{DD}}\left(\left[n_{\lambda}\right] ; \mathbf{r}\right)=v_{\mathrm{x}}^{\mathrm{DD}}([n] ; \lambda \mathbf{r}),
$$

which is a contradiction to Eq. (B7).

We specifically note in passing that the correction of Cerqueira et al. [70],

$$
E_{\mathrm{x}}^{\mathrm{AK} 13,0}=E_{\mathrm{x}}^{\mathrm{AK} 13}+v_{\mathrm{x}}^{\mathrm{DD}} \int n(\mathbf{r}) d^{3} r,
$$


is not the energy functional corresponding to the realigned potential of Eq. (13) (as one can show using Janak's theorem in the spirit of Fig. 1) and would imply a discontinuity of the total energy $E(N)$ rather than of its derivative $\partial E / \partial N$.
[1] P. Hohenberg and W. Kohn, Phys. Rev. 136, B864 (1964).

[2] W. Kohn and L. J. Sham, Phys. Rev. 140, A1133 (1965).

[3] J. P. Perdew and K. Schmidt, AIP Conf. Proc. 577, 1 (2001).

[4] J. P. Perdew, A. Ruzsinszky, J. Tao, V. N. Staroverov, G. E. Scuseria, and G. I. Csonka, J. Chem. Phys. 123, 062201 (2005).

[5] P. Bleiziffer, A. Heßelmann, and A. Görling, J. Chem. Phys. 139, 084113 (2013).

[6] C. J. Umrigar and X. Gonze, Phys. Rev. A 50, 3827 (1994).

[7] M. J. Allen and D. Tozer, Mol. Phys. 100, 433 (2002).

[8] M. Lein and S. Kümmel, Phys. Rev. Lett. 94, 143003 (2005).

[9] R. Cuevas-Saavedra and V. N. Staroverov, Mol. Phys. 114, 1050 (2016).

[10] J. D. Ramsden and R. W. Godby, Phys. Rev. Lett. 109, 036402 (2012).

[11] S. V. Kohut, I. G. Ryabinkin, and V. N. Staroverov, J. Chem. Phys. 140, 18A535 (2014).

[12] I. Grabowski, E. Fabiano, A. M. Teale, S. Smiga, A. Buksztel, and F. D. Sala, J. Chem. Phys. 141, 024113 (2014).

[13] P. Verma and R. J. Bartlett, J. Chem. Phys. 140, 18A534 (2014).

[14] O. V. Gritsenko, L. M. Mentel, and E. J. Baerends, J. Chem. Phys. 144, 204114 (2016).

[15] S. Smiga, F. Della Sala, A. Buksztel, I. Grabowski, and E. Fabiano, J. Comput. Chem. 37, 2081 (2016).

[16] J. P. Perdew, R. G. Parr, M. Levy, and J. L. Balduz, Phys. Rev. Lett. 49, 1691 (1982).

[17] M. Mundt and S. Kümmel, Phys. Rev. Lett. 95, 203004 (2005).

[18] M. Thiele, E. K. U. Gross, and S. Kümmel, Phys. Rev. Lett. 100, 153004 (2008).

[19] M. Hellgren and E. K. U. Gross, Phys. Rev. A 85, 022514 (2012).

[20] P. Elliott, J. I. Fuks, A. Rubio, and N. T. Maitra, Phys. Rev. Lett. 109, 266404 (2012).

[21] T. Dimitrov, H. Appel, J. I. Fuks, and A. Rubio, New J. Phys. 18, 083004 (2016).

[22] S. V. Kohut, A. M. Polgar, and V. N. Staroverov, Phys. Chem. Chem. Phys. 18, 20938 (2016).

[23] N. Maitra, J. Chem. Phys. 144, 220901 (2016).

[24] D. Hofmann and S. Kümmel, Phys. Rev. B 86, 201109 (2012).

[25] F. Della Sala and A. Görling, Phys. Rev. Lett. 89, 033003 (2002).

[26] F. Della Sala and A. Gorling, J. Chem. Phys. 116, 5374 (2002).

[27] S. Kümmel and J. P. Perdew, Phys. Rev. Lett. 90, 043004 (2003).

[28] S. Kümmel and J. P. Perdew, Phys. Rev. B 68, 035103 (2003).

[29] P. Gori-Giorgi, T. Gál, and E. J. Baerends, Mol. Phys. 114, 1086 (2016).

[30] D. J. Tozer, J. Chem. Phys. 119, 12697 (2003).

[31] S. Kümmel, L. Kronik, and J. P. Perdew, Phys. Rev. Lett. 93, 213002 (2004).

[32] F. Wilken and D. Bauer, Phys. Rev. Lett. 97, 203001 (2006).

[33] S. R. Whittleton, X. A. S. Vazquez, C. M. Isborn, and E. R. Johnson, J. Chem. Phys. 142, 184106 (2015).

[34] A. D. Becke and M. R. Roussel, Phys. Rev. A 39, 3761 (1989).
[35] R. van Leeuwen and E. J. Baerends, Phys. Rev. A 49, 2421 (1994).

[36] A. D. Becke and E. R. Johnson, J. Chem. Phys. 124, 221101 (2006).

[37] A. P. Gaiduk and V. N. Staroverov, J. Chem. Phys. 128, 204101 (2008).

[38] V. N. Staroverov, J. Chem. Phys. 129, 134103 (2008).

[39] R. Armiento, S. Kümmel, and T. Körzdörfer, Phys. Rev. B 77, 165106 (2008).

[40] F. Tran, P. Blaha, M. Betzinger, and S. Blügel, Phys. Rev. B 91, 165121 (2015).

[41] E. Räsänen, S. Pittalis, and C. R. Proetto, J. Chem. Phys. 132, 044112 (2010).

[42] F. Tran, P. Blaha, and K. Schwarz, J. Chem. Theory Comput. 11, 4717 (2015).

[43] J. D. Gledhill and D. J. Tozer, J. Chem. Phys. 143, 024104 (2015).

[44] J. Carmona-Espindola, J. L. Gázquez, A. Vela, and S. B. Trickey, J. Chem. Phys. 142, 054105 (2015).

[45] F. Tran, P. Blaha, M. Betzinger, and S. Blügel, Phys. Rev. B 94, 165149 (2016).

[46] O. Gritsenko, R. van Leeuwen, E. van Lenthe, and E. J. Baerends, Phys. Rev. A 51, 1944 (1995).

[47] M. Kuisma, J. Ojanen, J. Enkovaara, and T. T. Rantala, Phys. Rev. B 82, 115106 (2010).

[48] F. Tran and P. Blaha, Phys. Rev. Lett. 102, 226401 (2009).

[49] D. Koller, F. Tran, and P. Blaha, Phys. Rev. B 85, 155109 (2012).

[50] F. Tran, P. Blaha, and K. Schwarz, J. Phys.: Condens. Matter 19, 196208 (2007).

[51] A. P. Gaiduk and V. N. Staroverov, J. Chem. Phys. 131, 044107 (2009).

[52] A. Karolewski, R. Armiento, and S. Kümmel, J. Chem. Theory Comput. 5, 712 (2009)

[53] M. Mundt, S. Kümmel, R. van Leeuwen, and P.-G. Reinhard, Phys. Rev. A 75, 050501(R) (2007).

[54] A. Karolewski, R. Armiento, and S. Kümmel, Phys. Rev. A 88, 052519 (2013).

[55] R. Armiento and S. Kümmel, Phys. Rev. Lett. 111, 036402 (2013)

[56] J. P. Perdew and Y. Wang, Phys. Rev. B 33, 8800 (1986).

[57] J. C. Slater, Phys. Rev. 81, 385 (1951).

[58] T. Kreibich, S. Kurth, T. Grabo, and E. K. U. Gross, Adv. Quantum Chem. 33, 31 (1998).

[59] M. Levy, J. P. Perdew, and V. Sahni, Phys. Rev. A 30, 2745 (1984).

[60] V. Vlček, G. Steinle-Neumann, L. Leppert, R. Armiento, and S. Kümmel, Phys. Rev. B 91, 035107 (2015).

[61] S. Kümmel and L. Kronik, Rev. Mod. Phys. 80, 3 (2008).

[62] C.-O. Almbladh and U. von Barth, Phys. Rev. B 31, 3231 (1985).

[63] G. Zhang and C. B. Musgrave, J. Phys. Chem. A 111, 1554 (2007). 
[64] J. D. Talman and W. F. Shadwick, Phys. Rev. A 14, 36 (1976).

[65] J. F. Janak, Phys. Rev. B 18, 7165 (1978).

[66] References [100,101] discuss that when going from integer to fractional particle numbers, there are different options for how to generalize a given functional to the ensemble case. Here we use the common definition and insert the density that integrates a fractional electron number into the regular functional expression.

[67] J. B. Krieger, Y. Li, and G. J. Iafrate, Phys. Rev. A 46, 5453 (1992).

[68] T. Aschebrock, R. Armiento, and S. Kümmel, Phys. Rev. B 95, 245118 (2017).

[69] A. Makmal, S. Kümmel, and L. Kronik, J. Chem. Theory Comput. 5, 1731 (2009).

[70] T. F. T. Cerqueira, M. J. T. Oliveira, and M. A. L. Marques, J. Chem. Theory Comput. 10, 5625 (2014).

[71] J. P. Perdew, K. Burke, and M. Ernzerhof, Phys. Rev. Lett. 77, 3865 (1996).

[72] A. Lindmaa and R. Armiento, Phys. Rev. B 94, 155143 (2016).

[73] A. D. Becke, Phys. Rev. A 38, 3098 (1988).

[74] While this is the case for LDA and EXX in the KLI approximation, for EXX (OEP) the orbital in question moves up in the energetic order from fifth- to third-highest occupied orbital.

[75] S. J. A. van Gisbergen, P. R. T. Schipper, O. V. Gritsenko, E. J. Baerends, J. G. Snijders, B. Champagne, and B. Kirtman, Phys. Rev. Lett. 83, 694 (1999).

[76] S. Kümmel and L. Kronik, Comput. Mater. Sci. 35, 321 (2006).

[77] B. Champagne, D. H. Mosley, M. Vračko, and J.-M. André, Phys. Rev. A 52, 178 (1995).

[78] M. Gruning, O. V. Gritsenko, and E. J. Baerends, J. Chem. Phys. 116, 6435 (2002).

[79] M. van Faassen, P. L. de Boeij, R. van Leeuwen, J. A. Berger, and J. G. Snijders, Phys. Rev. Lett. 88, 186401 (2002).

[80] P. Mori-Sánchez, Q. Wu, and W. Yang, J. Chem. Phys. 119, 11001 (2003).
[81] P. Umari, A. J. Willamson, G. Galli, and N. Marzari, Phys. Rev. Lett. 95, 207602 (2005)

[82] N. T. Maitra and M. van Faassen, J. Chem. Phys. 126, 191106 (2007).

[83] T. Körzdörfer, M. Mundt, and S. Kümmel, Phys. Rev. Lett. 100, 133004 (2008).

[84] A. Ruzsinszky, J. P. Perdew, G. I. Csonka, G. E. Scuseria, and O. A. Vydrov, Phys. Rev. A 77, 060502(R) (2008).

[85] C. D. Pemmaraju, S. Sanvito, and K. Burke, Phys. Rev. B 77, 121204(R) (2008).

[86] B. Champagne and B. Kirtman, Int. J. Quantum Chem. 109, 3103 (2009)

[87] T. Körzdörfer and S. Kümmel, in Theoretical and Computational Developments in Modern Density Functional Theory, edited by A. K. Roy (Nova Science, New York, 2012), pp. 211-222.

[88] L. Kronik, A. Makmal, M. L. Tiago, M. M. G. Alemany, M. Jain, X. Huang, Y. Saad, and J. R. Chelikowsky, Phys. Status Solidi B 243, 1063 (2006).

[89] F. Gygi, Phys. Rev. B 48, 11692 (1993).

[90] F. Hofmann (unpublished).

[91] J. R. Chelikowsky, N. Troullier, and Y. Saad, Phys. Rev. Lett. 72, 1240 (1994).

[92] F. Calvayrac, P.-G. Reinhard, E. Suraud, and C. A. Ullrich, Phys. Rep. 337, 493 (2000).

[93] M. A. L. Marques, A. Castro, G. F. Bertsch, and A. Rubio, Comput. Phys. Commun. 151, 60 (2003).

[94] S. Kümmel, J. Comput. Phys. 201, 333 (2004).

[95] J. C. Slater, Phys. Rev. 36, 57 (1930).

[96] S. F. Boys, Proc. R. Soc. London A 200, 542 (1950).

[97] R. McWeeny, Nature (London) 166, 21 (1950).

[98] F. G. Eich and M. Hellgren, J. Chem. Phys. 141, 224107 (2014).

[99] G. L. Oliver and J. P. Perdew, Phys. Rev. A 20, 397 (1979).

[100] E. Kraisler and L. Kronik, Phys. Rev. Lett. 110, 126403 (2013).

[101] A. Görling, Phys. Rev. B 91, 245120 (2015). 